\title{
Dez anos de um CAPSi: comparação da caracterização de usuários atendidos
}

\author{
lagor Brum Leitão ${ }^{a *}$ \\ Andréa Barbosa Dias ${ }^{\mathrm{a}}$ (1) \\ Kelly Guimarães Tristão ${ }^{b}$ (1) \\ Juliana Peterle Ronchia ${ }^{\text {(1) }}$ \\ Luziane Zacché Avellar ${ }^{\mathrm{a}}$ (1) \\ a Universidade Federal do Espírito Santo. Vitória, ES, Brasil \\ ${ }^{b}$ Faculdades Integradas São Pedro. Vitória, ES, Brasil
}

\begin{abstract}
Resumo: Estudo retrospectivo de caráter descritivo. Por meio de coleta em prontuários, objetivou-se caracterizar crianças e adolescentes atendidos em um Centro de Atenção Psicossocial Infantojuvenil (CAPSi) durante seu décimo ano de funcionamento e comparar com estudo análogo realizado no primeiro ano de funcionamento. Os dados foram analisados com auxílio dos softwares SPSS e Iramuteq. Identificou-se aumento de $51,85 \%$ ( $n=84)$ da demanda em relação ao primeiro ano. Observou-se aumento de usuários de sexo feminino, cuja marca foram queixas de automutilação. Manteve-se prevalência de usuários do sexo masculino, cuja marca foram problemas de comportamento, como agressividade e hiperatividade. Discute-se, neste estudo, as articulações entre CAPSi e demais serviços da Rede de Atenção Psicossocial (RAPS), especialmente acerca dos encaminhamentos. Também se discute sobre a medicalização sem registro de hipótese diagnóstica e os desafios de se exercer a clínica ampliada e a lógica do trabalho psicossocial, a qual se opõe ao paradigma exclusivamente biomédico, simplificador dos fenômenos do desenvolvimento infantil.
\end{abstract}

Palavras-chave: saúde mental, serviços de saúde, criança, adolescente.

\section{Introdução}

A Reforma Psiquiátrica Brasileira tem como principal direcionamento a oposição à lógica manicomial pautada numa clínica biomédica e excludente, implicando a necessidade de construção de outras formas de atuação. Nessa direção, a construção da clínica na reforma psiquiátrica deve ser orientada pela compreensão psicossocial do sofrimento psíquico, condição que circunscreve as práticas clínicas no âmbito de um cuidado muito mais amplo (Amarante, 1995). Isso significa dizer que todas as práticas clínicas devem implicar romper com as tendências normalizadoras, as quais tomam a patologia como objeto da clínica em detrimento do sujeito e da saúde como processo e expressão dos determinantes sociais (Scarcelli \& Alencar, 2009). Dito de outro modo, a clínica deve extrapolar o sentido meramente técnico do "tratar", de forma que inclua nesse espectro "técnico" cuidar, acolher, escutar, possibilitar ações emancipatórias e potencializar a qualidade de vida dos sujeitos (Ministério da Saúde, 2014a).

Após aprovação da Lei Federal no 10.216 (conhecida como "Lei Paulo Delgado"), que dispõe sobre a proteção e os direitos das pessoas com sofrimento psíquico, o modelo hospitalocêntrico passa ser substituído por uma

* Endereço para correspondência: leitao.iagor@hotmail.com rede de atenção, que além de considerar a liberdade e o acesso à cidadania a seus usuários, também deve corresponder aos princípios doutrinários do Sistema Único de Saúde (SUS). A proposta terapêutica passa a ser pautada na atenção e no cuidado psicossocial cotidiano, devendo o cuidado em saúde estar intimamente ligado às práticas de acolhimento, vínculo e corresponsabilização (Silva Júnior, Merhy \& Carvalho, 2003).

Nesse contexto, é atribuído ao Centro de Atenção Psicossocial (CAPS) função estratégica dentro dessa rede substitutiva. Além de acolher pessoas em sofrimento psíquico segundo a lógica psicossocial, os CAPS também passam a assumir papel político de articulador da rede de saúde, aproximando questões relativas à saúde coletiva e à saúde mental no intuito de suscitar um campo interdisciplinar de saberes e práticas (Furtado \& Onocko-Campos, 2006).

No entanto há que se destacar que os CAPS, inicialmente, não compreenderam todos os segmentos da população em sofrimento psíquico. Por muito tempo, crianças e adolescentes em sofrimento psíquico grave, severo e persistente estiveram às margens das políticas públicas de saúde. Para Couto, Duarte e Delgado (2008), as políticas de saúde mental da criança e do adolescente (SMCA), norteadas pelos princípios da Reforma Psiquiátrica, deram-se tardiamente no Brasil. 
Somente em 2002 foi instituída a Portaria no 336/2002, que estabelece, dentre outras modalidades, o Centro de Atenção Psicossocial Infantojuvenil (CAPSi), serviço direcionado à atenção específica da população infantojuvenil em sofrimento psíquico grave, severo e persistente ("Portaria n ${ }^{\circ} 336$ ", 2002). Vale esclarecer que esse serviço foi instituído dezesseis anos depois da abertura do primeiro CAPS, inaugurado na cidade de São Paulo no final de 1986. Pode-se falar, portanto, de uma dívida histórica do movimento da Reforma Psiquiátrica no que tange ao público infantojuvenil (Cirino, 2004).

Couto et al. (2008) destacam quatro fatores que contribuíram para a inclusão tardia do público infantojuvenil na agenda das políticas de saúde mental do Brasil. São eles: (1) a complexidade da avaliação diagnóstica que se relaciona a esse campo; (2) carência de estudos que considerem a especificidade de fatores regionais/culturais na proteção ou risco à saúde mental de crianças e adolescentes; (3) ausência de evidências consistentes sobre a eficácia de tratamentos voltados para esse público; e (4) a dificuldade de articulação efetiva entre os membros da rede que compõe direta e indiretamente a assistência (setores da saúde, setor educacional, assistencial e jurídico, por exemplo).

A existência dos CAPSi não apenas deu um lugar possível de cuidado para esse público como também deu maior visibilidade ao sofrimento psíquico na infância e adolescência, questão que por muito tempo foi ignorada pelo campo da saúde pública (Reis, Delfini, Dombi-Barbosa, \& Oliveira, 2010). Inclusive, foi a partir da implementação dos CAPSi que o Ministério da Saúde passou a produzir as bases, princípios e diretrizes de uma política pública de saúde mental dirigida a crianças e adolescentes.

As diretrizes ministeriais (Ministério da Saúde, 2005; 2014a; 2014b; "Portaria $n^{\circ} 3.088$ ", 2011) sugerem que os cuidados em SMCA se desenvolvam em diversos serviços de saúde, que vão desde a atenção básica - Unidade Básica de Saúde (UBS), Estratégia Saúde da Família (ESF), Núcleo de Apoio à Saúde da Família (NASF), Consultório na Rua e Centro de Convivência e Cooperativa (CECCO) à atenção secundária (CAPSi, ambulatórios e hospitais gerais). Embora o CAPSi figure uma modalidade de serviço dentre outros, nele situam-se valores clínico-políticos que são singulares, na medida que se pressupõe um espaço e uma equipe interdisciplinar mais bem capacitada para $o$ cuidado de casos que envolvem sofrimento psíquico grave, severo e persistente.

Ao figurar um espaço que corporifica a lógica do cuidado em liberdade, cotidiano e território, o que na política brasileira é nomeada de "atenção psicossocial", o CAPSi mostra-se como objeto de pesquisa privilegiado para o desenvolvimento de análises críticas sobre os impasses existentes no empreendimento de ações psicossociais destinadas à infância e adolescência. Sobre esse serviço, a literatura tem destacado as seguintes dificuldades: (1) a precarização do trabalho e a má gestão nos diferentes níveis de governo; (2) pouca capacitação dos profissionais para o trabalho em SMCA, especialmente no que diz respeito às referências técnicas para o cuidado de usuários com transtorno do espectro autista (TEA) e psicoses; (3) desconhecimento da população sobre os serviços oferecidos pelos CAPSi; (4) pouco investimento em apoio matricial e práticas intersetoriais incipientes; (5) alto índice de crianças e adolescentes encaminhados por queixas de dificuldades no espaço escolar-educacional; e (6) a dificuldade das equipes dos CAPSi constituírem "lugar" possível para o cuidado de crianças e adolescentes com problemas ligados ao uso de álcool e outras drogas conforme os pressupostos da lógica psicossocial e da política da redução de danos (Carvalho, Gondim, Holanda, \& Alencar, 2014; Couto \& Delgado, 2015; Monteiro et al., 2012; Muylaert, Delfini, \& Reis, 2015; Ronchi \& Avellar, 2010; Salvador \& Pio, 2016; Teixeira \& Jucá, 2014; Tristão, 2018).

No intuito de contribuir para o campo da SMCA, este estudo objetivou caracterizar as crianças e os adolescentes atendidos em um CAPSi durante seu décimo ano de funcionamento (2016-2017) e comparar com estudo análogo realizado no primeiro ano de funcionamento (2006-2007) (Ronchi \& Avellar, 2010). Aposta-se que a comparação pode contribuir com indicadores para a adequação das políticas em SMCA à realidade local desse serviço, além de refletir se as suas práticas estão em consonância com as propostas e diretrizes ministeriais e com o que tem sido discutido pela literatura sobre os CAPSi.

\section{Método}

Estudo documental de caráter retrospectivo e descritivo. Acessou-se prontuários eletrônicos de crianças e adolescentes que receberam algum tipo de atendimento no espaço físico do CAPSi, entre setembro de 2016 e setembro de 2017, com o intuito de coletar dados como: (1) idade; (2) sexo; (3) escolaridade; (4) tempo em que o usuário ficou sob os cuidados do CAPSi; (5) fonte de encaminhamento para o CAPSi; (6) queixa que motivou a procura de cuidado no CAPSi; (7) diagnósticos; e (8) uso de medicamentos. Tais informações foram transcritas e organizadas uma planilha apropriada.

Em seguida, os dados obtidos dos prontuários foram submetidos à análise estatística por meio do Statistical Package for the Social Sciences (SPSS), versão 19.0 para Windows. Por tratar-se de pesquisa descritiva, verificou-se apenas a frequência da ocorrência das variáveis. Optou-se, no decorrer do estudo, utilizar o recurso "Nuvem de palavras" do software Interface de R pour les Analyses Multidimensionnelles de Textes et de Questionnaires (Iramuteq). A Nuvem foi utilizada para contabilizar e ilustrar as principais queixas (resultado da avaliação dos profissionais sobre os problemas apresentados por quem demandou o serviço no momento do acolhimento) registradas nos prontuários dos usuários. Embora o SPSS possibilite contabilizar palavras por frequência, o recurso da Nuvem do Iramuteq possibilita agrupar e organizar graficamente as palavras de acordo com a frequência, permitindo facilmente uma visão panorâmica (Camargo \& Justo, 2013). 


\section{Resultados}

A Tabela 1 ilustra as características sociodemográficas dos usuários globais (todas crianças e adolescentes que receberam algum tipo de atendimento pelo CAPSi no recorte de tempo estabelecido pelo estudo). Notou-se maiores prevalências de adolescentes no serviço $(65,03 \% ; n=160)$ e de usuários do sexo masculino $(64,63 \%$; $\mathrm{n}=159$ ). No que se refere aos níveis de escolaridade, a maioria dos usuários $(43,5 \% ; n=107)$ está no nível fundamental. Chama atenção a parcela significativa de prontuários que não constam as informações de escolaridade dos usuários $(25,65 \%$; $n=63)$ e de usuários que recebem auxílio do Bolsa Família (42,27\%; n= 104).

A Tabela 2 apresenta as portas de entrada dos usuários para o serviço do CAPSi. Notou-se que a maioria foi encaminhada pelas UBS. As demandas espontâneas ficaram em segundo lugar. Novamente identificou-se porcentagem significativa de prontuários $(22,35 \%)$ que não continham informações sobre a procedência dos usuários.

Tabela 1. Características sociodemográficas das crianças e adolescentes atendidos pelo CAPSij entre Setembro de 2016 e Setembro de 2017

\begin{tabular}{lcc}
\hline Características sociodemográficas & $n$ & $\%$ \\
\hline Total de usuários & 246 & 100 \\
Faixas etárias & - & - \\
$1-5$ anos & 32 & 13 \\
$5-10$ anos & 54 & 21,9 \\
$11-15$ anos & 87 & 35,36 \\
$16-18$ anos & 73 & 29,67 \\
Sexo & - & - \\
Feminino & 87 & 35,36 \\
Masculino & 159 & 64,63 \\
Cor & - & - \\
Branca & 58 & 23,57 \\
Negra & 21 & 8,53 \\
Parda & 90 & 36,58 \\
Não consta & 77 & 31,3 \\
Auxílio Bolsa Família & 104 & 42,27 \\
Escolaridade & - & - \\
CMEI & 3 & 1,21 \\
Creche & 22 & 8,94 \\
Ensino Fundamental & 107 & 43,5 \\
Ensino Médio & 36 & 14,63 \\
EJA & 2 & 0,81 \\
Não frequenta & 13 & 5,28 \\
Não consta & 63 & 25,65 \\
\hline
\end{tabular}

Tabela 2. Portas de entrada para o CAPSij

\begin{tabular}{cccccccccc}
\hline APAE & $\begin{array}{c}\text { Conselho } \\
\text { tutelar }\end{array}$ & Creas & $\begin{array}{c}\text { Demanda } \\
\text { espontânea }\end{array}$ & Escola & Hospital & $\begin{array}{c}\text { Unidade Básica } \\
\text { de Saúde }\end{array}$ & $\begin{array}{c}\text { Varada } \\
\text { Infância }\end{array}$ & Outros & Não consta \\
\hline $\mathrm{n}=10$ & $\mathrm{n}=16$ & $\mathrm{n}=9$ & $\mathrm{n}=23$ & $\mathrm{n}=15$ & $\mathrm{n}=11$ & $\mathrm{n}=95$ & $\mathrm{n}=4$ & $\mathrm{n}=8$ & $\mathrm{n}=55$ \\
$4 \%$ & $6,5 \%$ & $3,65 \%$ & $9,34 \%$ & $6 \%$ & $4,5 \%$ & $38,16 \%$ & $1,62 \%$ & $3,25 \%$ & $22,35 \%$ \\
\hline
\end{tabular}

$n$ : valor absoluto.

No que se refere ao tempo em que os usuários ficaram sob os cuidados do CAPSi, notou-se que $27,23 \%(n=67)$ foram atendidos uma ou duas vezes pela a equipe do centro. Na maioria dos prontuários desses usuários havia informações sobre tentativas de ligações dos profissionais para as famílias, cujo objetivo era convocá-las para o serviço. No entanto, em muitos dos casos as ligações não foram retornadas e 
os usuários não retornaram - ao menos até o momento desta pesquisa. Em alguns prontuários dizia-se que o caso de determinado usuário não se configurava como "caso para CAPSi", o que gerava a contrarreferência do caso, isto é, o reencaminhamento para uma unidade de menor complexidade para dar seguimento ao cuidado.

Constatou-se que a Associação de Pais e Amigos dos Excepcionais (Apae) e as UBS foram as instituições mais visadas para o contrarreferenciamento. O principal motivo de encaminhar para as UBS foi o entendimento de que determinada criança ou adolescente demandava, naquele momento, somente a psicoterapia, não havendo portanto necessidade de acompanhamento por uma equipe de média complexidade como a do CAPSi. Já no que diz respeito à Apae, o principal motivo residiu no fato de ter sido identificado, nesses usuários, algum tipo de limitação cognitiva, como o retardo mental, não havendo portanto outro quadro psicopatológico associado e/ou comportamentos considerados "difíceis", como impulsividades heteroagressivas ou autoagressivas. É válido destacar que $36,25 \%(\mathrm{n}=24)$ de todos os usuários contrarreferenciados haviam chegado ao CAPSi por encaminhamento das UBS.

Buscou-se identificar nos prontuários as principais queixas registradas pelos profissionais no(s) primeiro(s) acolhimento(s) das crianças e adolescentes. Com o intuito de visualizar possíveis diferenças entre sexos, gerou-se duas nuvens de palavras, uma para as principais queixas registradas às meninas (Figura 1) e uma para as principais queixas registradas aos meninos (Figura 2). Observou-se que na nuvem das meninas houve prevalência de "automutilação", ao passo que na nuvem dos meninos a prevalência foi de "agressividade". As queixas ligadas às dificuldades nos contextos escolares estão presentes de forma significativa em ambos os sexos.

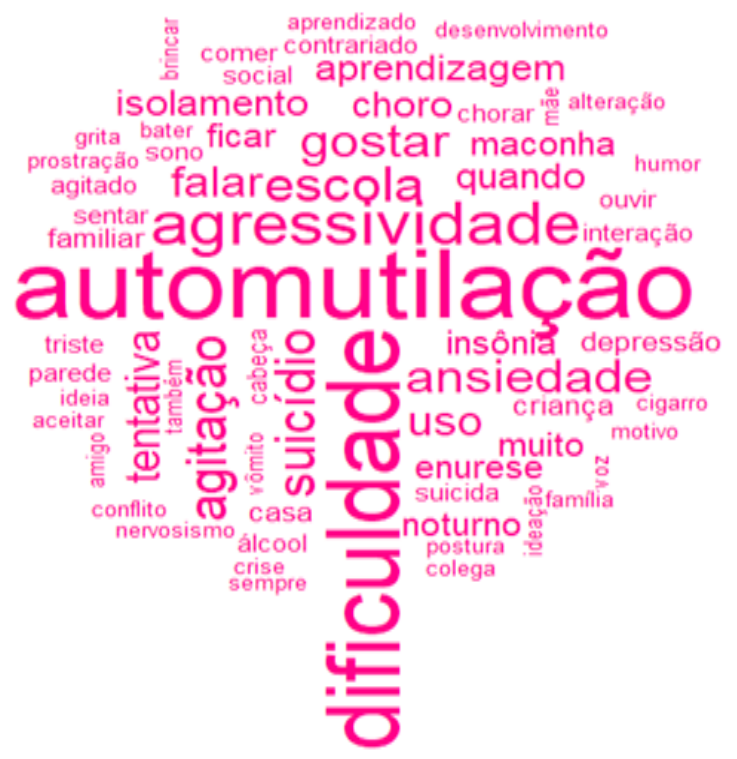

Figura 1. Nuvem de palavras referentes às queixas registradas no prontuário dos usuários do sexo feminino

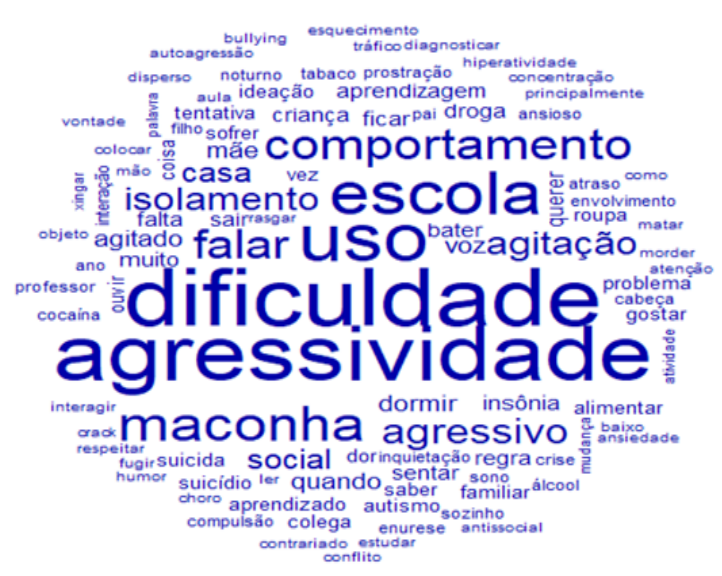

Figura 2. Nuvem de palavras referentes às queixas registradas no prontuário dos usuários do sexo masculino

\section{Dados sobre os usuários em atendimento regular pelo CAPSi}

Neste tópico são apresentados os dados referentes às crianças $\mathrm{e}$ adolescentes que estavam em atendimento regular $(\mathrm{n}=75)$ durante o recorte de tempo estabelecido pelo estudo. Observou-se parcela significativa de prontuários $(65,33 \% ; n=49)$ sem informações diagnósticas. Nos que continham, identificou-se os transtornos emocionais e de comportamento com início usualmente na infância e adolescência, o retardo mental e os transtornos do espectro autista como diagnósticos mais registrados. Esses quadros representaram uma parcela de $12 \%$ do total de diagnósticos.

Entre os 75 usuários ativos no serviço, identificou-se que 43 (57,33\%) fazem uso de medicação controlada. Entre os medicamentos mais utilizados destacaram-se risperidona, ritalina, e carbamazepina. Vale destacar que 17 desses usuários (39,53\%) utilizam medicamentos combinados.

\section{Caracterização dos usuários: houve mudanças?}

Com o intuito de comparar os resultados desta caracterização com os da caracterização de Ronchi e Avellar (2010), a Figura 3 ilustra, de forma sintetizada, os principais achados obtidos pelas duas caracterizações. Observou-se que o décimo ano de funcionamento do CAPSi foi marcado pelo aumento de 51,85\% $(n=84)$ na demanda em relação ao seu primeiro ano. Embora tenha ocorrido aumento de usuários do sexo feminino, os usuários do sexo masculino continuaram prevalentes. As UBS mantiveram-se como o serviço da rede que mais encaminha crianças e adolescentes para o CAPSi, seguidas pelas demandas espontâneas. Por fim, observou-se que as principais queixas daqueles que buscaram os serviços do CAPSi obtiveram pouca oscilação. A maior diferença residiu em maiores registros de queixas ligadas às dificuldades de atenção e aprendizagem e riscos de suicídio. 

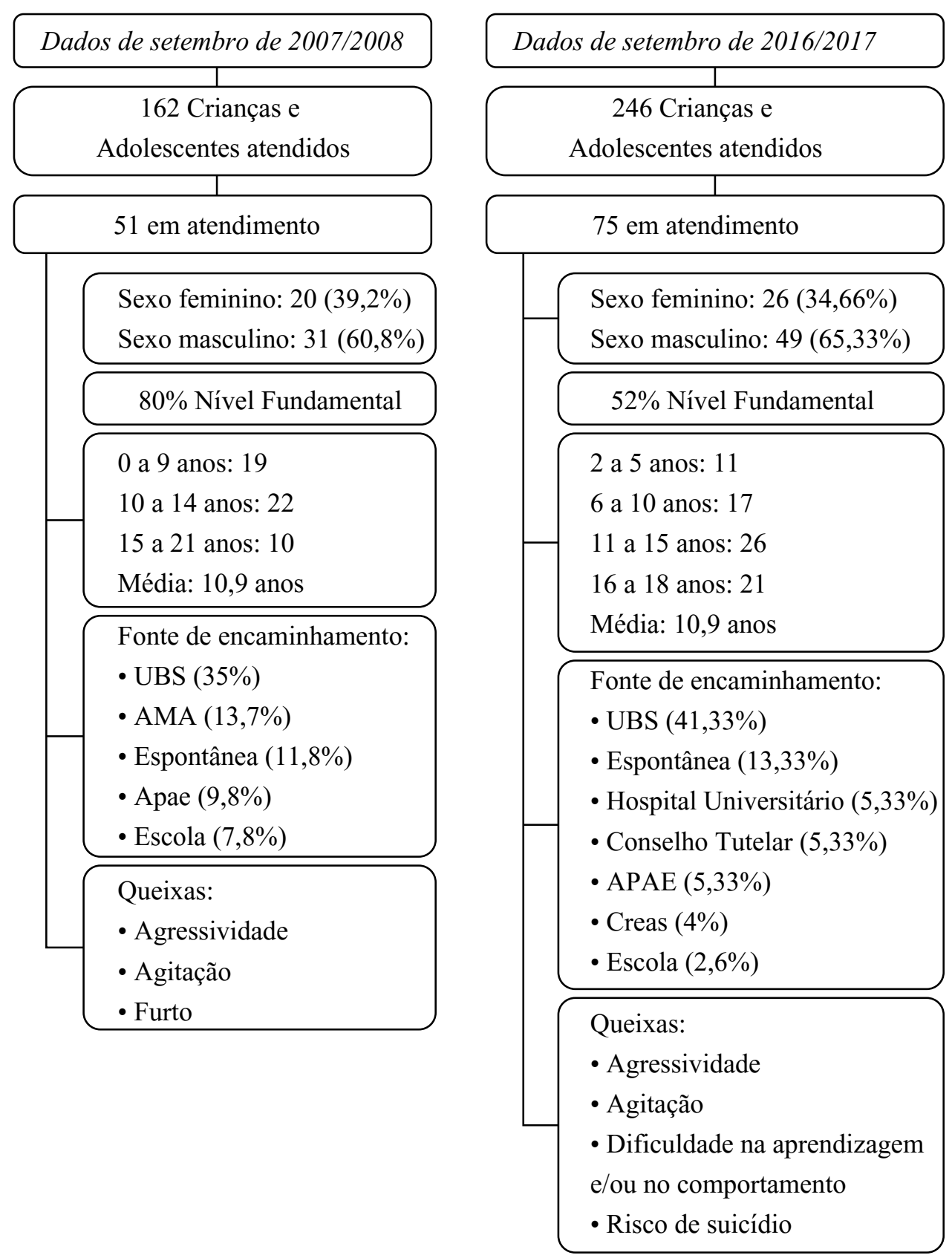

Figura 3. Comparação dos principais resultados referentes às duas caracterizações das crianças e adolescentes atendidos pelo CAPSij de Vitória

\section{Discussão}

\section{Acerca das prevalências}

A prevalência de usuários do sexo masculino nos serviços do CAPSi é unânime entre os estudos de caracterização. A taxa encontrada neste estudo (64,63\%) está em concordância com a literatura, que descreve taxas entre $60 \%$ e 70,3\% (Daltro, Moares, \& Marsiglia, 2018; Delfini, Dombi-Barbosa, Fonseca, Tavares, \& Reis, 2009; Hoffman, Santos, \& Mota, 2008; Ronchi \& Avellar, 2010). De acordo com Machado et al. (2014), o fato de os meninos apresentarem maiores problemas do tipo externalizantes (hiperatividade e agressividade, principalmente) faz com que eles tenham maior probabilidade de serem encaminhados aos serviços de saúde, na medida que esses problemas costumam causar maiores incômodos no laço social.

Em nosso estudo identificou-se que na nuvem das meninas (Figura 1) houve destaque para queixas de "automutilação", enquanto na nuvem dos meninos (Figura 2) o destaque foi para as queixas de "agressividade". Mas não seria a automutilação ela mesma uma agressividade? Entende-se que os desdobramentos 
dessa agressividade têm se dado de formas distintas entre os sexos. Os dados sugerem que os meninos tendem a expressar a "agressividade" de forma "exo", isto é, para fora, através de comportamentos agressivos para com o outro, enquanto as meninas tendem a expressá-las de uma formo "endo", para dentro, no apelo ao corpo e por meio das automutilações.

Em revisão de literatura sobre o comportamento autolesivo, Silva e Botti (2017) destacam que fatores psicopatológicos específicos, como transtornos depressivos, de ansiedade, personalidade borderline e psicoses tendem a figurar comportamentos de impulsividade e/ou sentimentos de baixa autoestima que estariam associados com a automutilação entre as adolescentes, especialmente nas faixas etárias de 15 a 17 anos. Entre esses fatores de risco para o comportamento autolesivo, os autores também chamam a atenção para os familiares (história de maltrato físico pelos cuidadores, separação precoce dos pais, abuso sexual e negligência emocional) e os sociais (isolamento social, bullying, dificuldades escolares, amigos com história de automutilação).

Ora, não estariam crianças e adolescentes em sofrimento psíquico, usuários de um CAPSi, circulando, em maior ou menor grau, pelas experiências descritas? Nesses termos, destaca-se a pertinência de se escutar a automutilação como uma via que ganha cada vez mais reconhecimento entre meninas como meio de expressão do sofrimento psíquico.

De acordo com o Ministério da Saúde (2010), o problema das automutilações em jovens tem se tornado questão de saúde pública, especialmente devido à tendência de correlação entre comportamento autolesivo e risco de suicídio. Parece-nos que é por essa via, o apelo ao corpo, que uma parcela significativa de meninas tem sido endereçada aos serviços de saúde mental, o que justificaria o aumento de adolescentes do sexo feminino no CAPSi estudado e o aumento de queixas que envolvem risco de suicídio. Para Bernardes (2015), casos de automutilação em crianças e adolescentes figuram experiências de sofrimento psíquico que denotam complexidade no cuidado, condição que situa o CAPSi como o serviço mais adequado para acolhimento e cuidado dessa demanda.

Ainda sobre as prevalências, conforme indicado, destacou-se parcela significativa de usuários que recebem o auxílio Bolsa Família $(42,27 \%$; n=104). Ressalta-se que a coleta desse dado só foi possível devido ao sistema on-line que interliga todos os equipamentos da rede municipal de saúde e assistência do município de Vitória, intitulado de "Rede Bem-Estar". Não foram encontrados estudos de caracterização que apresentam ou discutam esse dado. Destacamos que este dado configura pista para compreender o perfil de usuários dos CAPSi. Nesses serviços, são tomados para cuidado majoritariamente crianças e adolescentes provenientes de famílias com baixa renda. Não raro, encontram-se usuários em situações de extrema vulnerabilidade social, que habitam em laços familiares fragilizados, em abrigamentos ou ainda em contextos de vidas nas ruas (Delfini et al., 2009; Hoffman et al., 2008; Ronchi \& Avellar, 2010).

Nesse sentido, a prevalência de um público em vulnerabilidade social nos CAPSi é testemunha dos processos sócio-históricos de ações higienistas e institucionalizantes que marcaram a "saúde mental" no Brasil (Cervo \& Silva, 2014; Taño \& Matsukura, 2015). Quintanilha, Belotti, Tristão e Avellar (2017), em trabalho sobre a mesma unidade de CAPSi deste estudo, discutem a percepção dos profissionais sobre como o serviço costuma constituir um "porto seguro" para as crianças e adolescentes em vulnerabilidade social, "lugar no qual fogem da violência existente nos territórios em que moram" (p. 269). Acreditamos que o CAPSi, ao figurar lugar de cuidado básico (higiene, alimentação, segurança etc.) para com esses usuários, por exemplo, também permitem que eles encontrem algo para endereçarem. Trata-se de fazer desse momento de atender as necessidades um momento de cuidado e atendimento clínico, também. É sempre possível que algo ali se enderece, do nível da subjetividade e da demanda, e incidir sobre a demanda é responsabilidade daquele que dirige o cuidado, a equipe de saúde mental.

Nessa perspectiva, entende-se que "clínica" e "cuidado" não se separam; pelo contrário, ambas acontecem a partir das relações. "Entender que as diferentes demandas apresentadas ao serviço levam a diferentes formas de construção de cuidado, faz com que se estabeleçam clínicas, ou relações clínicas, também singulares" (Quintanilha et al., 2017, p. 270). Considerando que o CAPSi é um dos espaços públicos responsáveis pelo cuidado em saúde de crianças e adolescentes, cuidado orientado pelo paradigma da atenção psicossocial, entende-se que esse ato clínico (o cuidar) é essencialmente um ato político, posto que representa um modo do Estado democrático cuidar de seus cidadãos. Daí a necessidade de articulação entre os programas de saúde e de assistência.

\section{Portas de entrada para o CAPSi}

Conforme indicado pela Tabela 1, a maioria das crianças e adolescentes chegou ao CAPSi por encaminhamentos da UBS ( $\mathrm{n}=95 ; 38,16 \%)$, o que condiz com a Política Nacional de atenção básica. A política propõe que as portas de entrada do SUS sejam, prioritariamente, pela atenção básica, por meio das UBS e ESF, inclusive para os casos de saúde mental (Ministério da Saúde, 2013). Sobre este dado, em primeiro estudo sobre o supracitado CAPSi, Ronchi e Avellar (2010) verificaram que a equipe do serviço fazia reuniões frequentes com as UBS do município, orientando sobre o serviço prestado pelo CAPSi e a que população é dirigido, o que facilitaria o encaminhamento expressivo de pacientes por meio da atenção básica.

Conforme os resultados, observou-se parcela significativa de usuários $(27,23 \%)$ atendidos uma ou duas vezes pelo CAPSi. Nesta parcela estão inclusos 
aqueles que ainda não voltaram para o serviço e aqueles que não tiveram suas demandas entendidas como "caso para cuidado no CAPSi" e que foram, portanto, contrarreferenciados para unidades de menor complexidade. Conforme indicado, 36,25\% $(\mathrm{n}=24)$ dos usuários contrarreferenciados haviam chegado ao CAPSi por encaminhamento de UBS. Nesse sentido, entende-se que o dado de prevalência de encaminhamentos de crianças e adolescentes ao CAPSi por UBS não permite, isoladamente, afirmar que há boa articulação entre os equipamentos. Destaca-se a importância desses equipamentos aprimorarem suas relações, no intuito de ordenarem melhor a demanda em SMCA da região e traçarem estratégias de cuidado. O trabalho em conjunto com a atenção básica, além de proporcionar atenção integral, compartilhando a responsabilidade pela qualidade de saúde de um dado território, também tornam os dispositivos de atenção mais acessíveis àqueles que necessitam (Carvalho et al., 2014).

Ainda sobre esse tema, considerando que as ações em SCMA no contexto brasileiro foram historicamente marcadas por estratégias de segregação e institucionalização (Taño \& Matsukura, 2015), entendemos que pode persistir certa imagem de um "CAPSi-preceptor", cuja função seria receber toda e qualquer demanda que possa ser tipificada como "caso de saúde mental". Esse imaginário, produto de uma lógica biomédica e ortopédica, conduz profissionais e serviços de saúde a práticas que pouco se ocupam do sofrimento psíquico e que não valorizam a corresponsabilização do cuidado. Para Teixeira, Couto e Delgado (2017), as ações de saúde mental na atenção básica, ao constituírem uma rede ampliada, podem reduzir encaminhamentos desnecessários e aumentar a adesão de usuários e familiares ao tratamento. Os autores afirmam que quando as ações da atenção básica estão articuladas aos CAPSi, elas "tendem a promover fluxos assistenciais menos fragmentados, facilitando a continuidade da atenção, compartilhamento das estratégias de cuidado e aumento da efetividade da ação psicossocial" (Teixeira et al., 2017, p. 1934).

Ainda sobre o tema das portas de entrada, estudos têm demonstrado que parcela significativa de encaminhamentos aos CAPSi está relacionada às dificuldades nos contextos escolares (Beltrame \& Boarini, 2013; Delfini et al., 2009; Luckow \& Cordeiro, 2017). De acordo com Delfini et al. (2009), a escola tem sido crescentemente um espaço onde são evidenciadas e reconhecidas dificuldades de crianças e adolescentes em corresponderem a certo padrão de ideal, que vão desde às capacidades cognitivas e de produtividade escolar, às inadequações comportamentais no espaço escolar-institucional (comportamentos de rebeldia, agressividade, apatia etc.). Para Beltrame e Boarini (2013), dificuldades comportamentais como essas e o não êxito escolar acabam justificando a busca por parcerias em setores da saúde, especialmente àqueles com escopo voltado para a saúde mental, como o CAPSi. Em muitos casos, no entanto, o repensar das práticas escolares não é considerado neste processo, o que contribui para que as crianças e adolescentes sejam tomadas, de saída, como desviantes e tipificadas com transtornos psíquicos e/ou comportamentais.

Sobre esse tema, Hoffman et al. (2008) argumentam que a alta incidência de crianças e adolescentes com problemas relacionados às dificuldades nos contextos escolares nos CAPSi tendem a prejudicar o cuidado de casos que envolvem sofrimento psíquico intenso, especialmente as psicoses e autismos. Em nosso entendimento, o crescente endereçamento de sujeitos com queixas de dificuldades de aprendizagem e de comportamento nos espaços escolares-institucionais aos serviços de saúde mental, além de ser testemunha dos efeitos de práticas biomédicas e normativas, pode ser lido como um sintoma social. Além disso, é válido destacar que, em sentido último, não existe demanda incorreta ou irrelevante. No entanto, é preciso suscitar uma práxis de saúde nesses serviços de forma que promovam respostas mais responsáveis a essas demandas e impliquem todos aqueles que compõem funções subjetivas para determinado usuário. Nesse entendimento, cabe ao CAPSi desconstruir demandas como essas, suscitando a possibilidade de novos modelos de atuação à queixa escolar, quando se é endereçada a "resolubilidade" exclusivamente ao campo da saúde.

No que se refere ao CAPSi deste estudo, embora a parcela de encaminhamentos realizados por escolas tenha sido relativamente baixa $(6 \% ; n=15)$, identificou-se, conforme as Figuras 1 e 2, que as dificuldades nos contexto escolar estão entre as principais queixas que motivaram a busca por atendimento no CAPSi, seja para os meninos, seja para as meninas. Vale lembrar também que em muitos casos as escolas encaminham crianças e adolescentes para as UBS, e que, posteriormente, são encaminhadas ao CAPSi. Nesse sentido, aponta-se a importância de estudos futuros que investiguem e acompanhem, de forma mais aprofundada, o "itinerário" das crianças e adolescentes até chegarem ao CAPSi, especialmente aquelas circunscritas em queixas no âmbito escolar.

Por fim, chamou atenção o fato de as demandas espontâneas figurarem a segunda maior porta de entrada para os serviços do CAPSi. Embora o aumento possa ser tido como positivo, posto que indica maior conhecimento da população acerca das atividades do CAPSi, entende-se que a taxa de aumento (21,05\%) está aquém do esperado para um serviço que funciona há dez anos e cujas portas de entradas estão sempre abertas. Inclusive, todos os quantitativos da demanda, tanto a global $(n=246)$ quanto a que se refere aos usuários em atendimento regular $(\mathrm{n}=75)$, estão relativamente baixos tendo em vista: (1) o tempo de funcionamento do serviço; (2) o fato de que esse foi, desde sua inauguração até o ano 2017, o único CAPSi existente no estado do Espírito Santo; e (3) o fato do serviço funcionar com cerca de 19 profissionais que atuam diretamente no cuidado de crianças e adolescentes (assistentes sociais, arteterapeutas, 
enfermeiros e técnicos de enfermagem, médicos pediatras e psiquiatras, musicoterapeuta, psicólogos, terapeutas ocupacionais) (“Capsi é centro", 2019).

\section{Diagnósticos e medicalização}

Conforme indicado, constatou-se parcela expressiva de prontuários sem informações diagnósticas dos usuários em atendimento intensivo ou semi-intensivo $(65,33 \%$; $\mathrm{n}=49$ ). Embora se possa entender esse fato como efeito da dificuldade e complexidade de avaliação diagnóstica de psicopatologias na infância (Couto et al., 2008; Silva \& Ghazzi, 2016), muitos desses usuários fazem uso de medicamentos psiquiátricos, prescritos pelo corpo técnico do serviço. Entre os mais utilizados destacam-se os antipsicóticos, psicoestimulantes, antidepressivos e anticonvulsivantes. Dos usuários que fazem uso dessas medicações, somente $34,88 \%(n=15)$ possuíam diagnóstico. Diante desses dados interroga-se a prescrição de medicamentos como risperidona, fluoxetina e carbamazepina para crianças e adolescentes sem registros de hipóteses diagnósticas em seus prontuários.

Sobre essa questão Ronchi e Avellar (2010), em primeiro estudo, descreveram que a equipe desse CAPSi mostrava cuidado em diagnosticar as crianças e os adolescentes por entenderem que, em alguns casos, o diagnóstico em padrões fixos de comportamento poderia tamponar uma direção de tratamento que considere o desenvolvimento das relações do sujeito a estrutura e o meio ambiente. Todavia os dados atuais sugerem que se por um lado há cuidado em diagnosticar, não há, ao que parece, um cuidado em medicar, e mais: menos diagnósticos não figuraram menos medicação. Sobre esse aspecto, entendemos que medicar sem antes construir uma hipótese diagnóstica parece figurar um "critério operacional", cada vez mais adotado pelas nosografias atuais, de que é através da resposta à medicação que se pode formular uma hipótese diagnóstica.

Nessa perspectiva, subtraímos o quanto o modelo manicomial e prescritivo ainda permeia as práticas nos serviços de atenção psicossocial, na medida em que são respondidas demandas de normatização no intuito de tamponar o mal-estar que a infância produz no projeto social. Conforme indicado, tais práticas pouco se ocupam das experiências e narrativas de sofrimento psíquico. Nessa direção, fica claro que o rompimento com o modelo manicomial e biomédico vai além do fechamento dos hospitais psiquiátricos, posto que os muros manicomiais não são apenas físicos, mas culturais e ideológicos, ainda sustentados pela simplificação e pelo mal-uso dos saberes "psi”, os quais frequentemente reproduzem práticas engessadas e ortopédicas. Sobre esse tema, vamos de encontro à crítica de Alberti e Figueiredo (2006) sobre a clínica em saúde mental de caráter ortopédica, tida como antagônica ao modelo de atenção psicossocial: "administrar pílulas para pessoas com problemas psicológicos é absolutamente experimental, não faz participar o sujeito, que, assim, é desresponsabilizado do mal do qual se queixa, o que o faz de cobaia de uma experiência" (p. 12).

Considerando a lógica "psicossocial", as ações das equipes dos serviços de saúde, seja da atenção básica ou secundária, devem conformar um projeto terapêutico singular (PTS) para cada usuário (Ministério da Saúde, 2003). No caso do cuidado em SMCA, o PTS também deve ser elaborado pela equipe e, principalmente, corresponder à realidade de vida de determinada criança/adolescente e sua família. Nesse sentido, a medicação figura uma dentre inúmeras possibilidades de intervenção/tratamento com um usuário de saúde mental, embora em muitos casos seu uso seja empregado sobremaneira e ainda sem o devido trabalho do lugar simbólico do medicamento no processo de cuidado, particularmente em crianças e adolescentes (Carvalho, 2012; Silva, Guazina, Pizzinato, \& Rocha, 2019). A tendência de uma exclusividade terapêutica e, portanto, de uma especialidade profissional, é testemunha da não efetividade do trabalho interdisciplinar e da não circularização do saber - não há a clínica ampliada, portanto. Para Onocko-Campos (2001), não é raro encontrar um modus operandi nos serviços de saúde mental de caráter ortopédico que dissocia o usuário em diferentes objetos das especialidades: "Remédio é com psiquiatra. Escuta é com psicólogo. Trabalho é com terapeuta ocupacional. Intercorrência clínica, outra: não é conosco. Surto? Vai ter que internar" (p. 103).

Há algo muito próprio da clínica com crianças que pode interessar a essa discussão. Primeiro que a estrutura psíquica não se define no tempo da infância, o que, para Vanoli e Bernardino (2008), torna-se possível, mediante a escuta, "fazer advir um sujeito onde antes se acreditava estar instalado um objeto" (p. 250). Dito de outro modo, é possível pensar as grandes estruturas clínicas (neurose, perversão e psicose, especialmente) no tempo na infância como não-incididas. Essa possibilidade permite ao clínico trabalhar com o que Jerusalinksy (2018) chama de "risco". A autora entende que o mais importante diagnóstico para intervenção é o reconhecimento do "não está bem, precisa de ajuda". No entanto, isso não deve ser procedido de alguma correlação antecipatória por psicopatologia específica, posto que não é sobre a psicopatologia em si que o clínico atuará, mas sobre o sofrimento psíquico. Trabalhar única e exclusivamente sobre a psicopatologia implica tratar fenômenos humanos complexos a partir de categorias nosológicas, condição que fortalece o paradigma biomédico como simplificador dos fenômenos do desenvolvimento infantil (Lima, 2005).

Nesse sentido, entendemos que a ação de "entrar com a medicação" sem antes ter uma hipótese diagnóstica, em sentido amplo, não configura o "trabalhar com o risco", posto que aí se produz a antecipação psicopatológica. Se tivermos como fundamento os pressupostos da atenção psicossocial, deve-se, nesses casos, questionar qual o risco que a medicação irá intervir: o risco de atender a escola que não se conforma à criança/adolescente? 
O risco da família que angustiada por não saber mais o que fazer? Ou o risco de o próprio discurso do técnico não suportar um furo no saber, prescrevendo uma pílula sem antes situar sua função coletivamente diante a equipe multiprofissional e subjetivamente diante o usuário e seus cuidadores? Se não engendrarmos questionamentos como esses, corremos na verdade o risco da patologização de comportamentos cuja ação clínica não inclui o sujeito que dá sentido ao comportamento e ao sintoma por ele endereçado. A tendência, nesses casos, é a produção de um usuário dissociado do serviço e de um sujeito dissociado do seu cuidado.

Quando os serviços de saúde não figuram espaço de amparo e escuta, praticando o uso de psicofármacos como estratégia primeira de intervenção sobre o sofrimento infantil, desrespeitam a condição de sujeito da criança e do adolescente. Sobre esse tema, inclusive, destaca-se que a política de SMCA fortalece o entendimento de que só é possível exercer uma clínica efetiva com crianças e adolescentes se esta for situada no campo da ética.

Antes e primeiro que tudo, é preciso adotar como princípio a ideia de que a criança ou o adolescente a cuidar é um sujeito. Tal noção implica, imediatamente, a de responsabilidade: o sujeito criança ou adolescente é responsável por sua demanda, seu sofrimento, seu sintoma. E, por conseguinte, um sujeito de direitos, dentre os quais se situa o direito ao cuidado. (Ministério da Saúde, 2005, p. 11)

Se o documento coloca, de saída, uma ressalva deste tipo, é justamente porque aí reside uma das maiores dificuldades clínica: o responsabilizar-se para que a condição de sujeito daquele que está sendo cuidado seja preservada e tomada como motor do tratamento. Sobre esse tema, entendemos que algumas ressalvas devem ser feitas: crianças e adolescentes costumam ser encaminhadas à profissionais e serviços de saúde mental, públicos ou privados, por não corresponderem a uma norma compartilhada pelo laço social, isto é, por causarem uma ruptura com um certo padrão de ideal. Tomando como base uma perspectiva canguilheniana ${ }^{1}$, poder-se-ia dizer que elas são endereçadas à clínica em saúde mental devido à incapacidade de serem normativas, de responderem ao padrão valorativo e/ou estatístico. No entanto, o fato de um terceiro (familiar/cuidador, profissional e/ou instituição) visualizar um sintoma na criança ou adolescente e incomodar-se com ele, mostra-se frequentemente como condição inicial para que cheguem para atendimento; pois são eles, os "terceiros",

1 Em Onormal e o patológico, Canguilhem (1943/2002) critica dois critérios então utilizados para o estabelecimento do normal: o critério quantitativoestatístico e o critério qualitativo-normativo. As críticas de Canguilhem nos servem de bússola para orientarmos uma escuta clínica que não tome, de saída, comportamentos desviantes de uma norma como patológicos (transtornos em si), mas como um sinal de alerta que nos convoca à compreensão da história do indivíduo com a sua estrutura e meio ambiente. que demandam inicialmente avaliação e/ou tratamento (Faria, 2016; Onocko-Campos, 2001).

Quando diversos autores apontam que um dos maiores desafios da clínica em SMCA está ligado à complexidade diagnóstica (Couto et al., 2008; D’Abreu, 2012; Silva \& Ghazzi, 2016), apoiam-se no fato de que, toda criança em sofrimento psíquico, seja ele desencadeado pelos atrasos ou transtornos no desenvolvimento, ou ainda de conflitos familiares, em contextos escolares e/ou no território, transbordam nas relações sociais e na capacidade de responderem aos ideais. Logo, os comportamentos de agressividade, agitação, automutilação, dificuldades de aprendizagem, uso de drogas e isolamento (conforme ilustrado nas nuvens de palavras, Figura 1 e Figura 2) figurariam índice ou símbolo de sofrimento psíquico, o que faria com que esses sujeitos encontrassem um espaço possível no campo da saúde mental. No entanto, ao chegar para atendimento, é fundamental que o sintoma $n a$ criança/adolescente seja colocado entre parênteses, não devendo-o ser aceito em seu estado bruto, e isso não significa excluí-lo ou desconsiderá-lo, mas questioná-lo. Caso contrário, isso implicaria na prática de uma clínica que não promove ações emancipatórias, o que mortifica o mandato da atenção psicossocial (Onocko-Campos, 2001).

Nessa perspectiva, entende-se que para fazer valer a política da intersetorialidade, do compartilhamento em rede, da corresponsabilização do cuidado etc., ou seja, práticas que compõem a atenção psicossocial (Ministério da Saúde, 2014a), é fundamental situar a função éticoclínica nesses serviços. Se não se tem dimensão da função clínica do momento que, em política, nomeia-se como "acolhimento" - que, importante ressaltar, é sempre um tempo lógico, e não cronológico -, esse momento será reduzido à função de triagem, cuja ação é esvaziada de ato clínico. Do mesmo modo, se não se tem dimensão da função clínica do encaminhamento, ele estará fadado a ir de encontro ao que diz o jargão popular do campo da saúde: "fazer orientar o fluxo" - prática antagônica ao entendimento de corresponsabilização do cuidado. É no registro do ato que se pode fazer com o que está escrito na política adquira valor clínico. Entretanto, para que o ato ganhe estatuto de ato clínico, ele deve ser precedido de uma aposta, que é, afinal, a aposta de que todo sujeito possa encontrar algo no serviço para se endereçar. Aqui, vale-nos o entendimento da função do cuidado em SMCA efetuada por Avellar e Safra (2018). Por meio de uma leitura winiccottiana, esses autores afirmam que

nas diferentes situações cotidianas do serviço, observa-se que muitas vezes a criança e/ou adolescente busca funções que não puderam ser constituídas no tempo certo de seus processos de desenvolvimento. Pode ser que viva ali, pela primeira vez, uma experiência que não pôde acontecer em nenhum outro momento de sua existência. Nesta situação, o profissional do serviço é o outro que precisa reconhecer que o paciente está 
buscando uma interlocução para uma experiência que ele não consegue nomear, mas que faz parte de sua história. (p. 147)

Nesse entendimento, cabe ao CAPSi constituir um ambiente de saúde que não leve em conta apenas os aspectos físicos, mas também os aspectos subjetivos e sociais como produtores das atuações profissionais (Ronchi \& Avellar, 2013). Ao promover um espaço subjetivo como esse, torna-se possível para profissionais e CAPSi ocuparem funções para seus usuários. É por meio dessas funções, que serão singulares para cada sujeito, que o CAPSi poderá promover relações de cuidado singulares para cada caso.

\section{A dimensão clínico-política dos CAPSi}

Os estudos de revisão sobre o CAPSi têm evidenciado que a população que mais acessa o serviço, considerando a totalidade dos atendimentos, não é a população prioritariamente descrita na política de SMCA (crianças e adolescentes com sofrimento psíquico grave e persistente), mas a população de crianças e adolescentes com problemas relacionados à conduta e aprendizagem que não configuram, em muitos casos, transtornos invasivos do comportamento, transtornos no desenvolvimento, neuroses graves ou psicoses (Bueno, 2013; Leitão, Constantinidis, \& Avellar, 2019). Embora a práxis em saúde mental se sustente ao colocar entre parênteses o desenvolvimento strictu do organismo, orientando a escuta para o sujeito que dá um sentido a esse organismo, condição que permite a escuta do sofrimento em suas diversas experiências, há que se destacar uma dimensão política que aí está em jogo e que permite orientar essa práxis em prol de uma organização da oferta de cuidado, além de fazer valer o que se chama de clínica ampliada.

Nesse sentido, entendemos que endereçar demandas de (des)conduta e dificuldades de aprendizagem a um serviço de saúde mental de média complexidade, por exemplo, sem a devida problematização da demanda, implica circunscrever crianças e adolescentes em um campo de uma psicopatologia e prejudicar o acolhimento e tratamento dos "transtornos graves, severos e persistentes" (como escrito na Portaria n ${ }^{\circ} 336 / 2002$ que funda serviço), público a quem o CAPSi se destina, prioritariamente. Além disso, demandar do campo da saúde mental explicação e intervenção no comportamento considerado desviante implica retirar da discussão as reponsabilidades dos laços territoriais, familiares, escolares e políticos sobre as condições de vida de um sujeito.

Para Furtado e Onocko-Campos (2005), as dimensões clínicas e políticas dos serviços de saúde mental tendem a gerar tensionamentos cotidianos nas tomadas de ações. Os autores entendem que a clínica, em sentido último, diz respeito ao caso tomado em sua singularidade. A saúde mental, por outro lado, embarcaria as ações políticas e éticas orientadas não à singularidade individual, mas à singularidade de um certo grupo, no qual seus integrantes compartilhariam um denominador comum. Nesses termos, entende-se que o fazer em SMCA não se limita aos serviços do CAPSi, tanto em sua dimensão clínica quanto em sua dimensão política. No entanto, ao localizarmos o CAPSi como um espaço-referência da rede de cuidado, alguns apontamentos podem ser tecidos: (1) O fato de o CAPSi ser configurado com uma equipe interdisciplinar (enfermeiros e técnicos de enfermagem, médicos pediatras e psiquiatras, psicólogos, terapeutas ocupacionais, assistentes sociais, fonoaudiólogos, entre outros), os quais devem atuar a partir de um denominador comum (o cuidado em SMCA), faz com que o serviço se constitua como espaço-referência nas questões que envolvam cuidado e tratamento do sofrimento psíquico na infância e adolescência; e (2) embora o CAPSi seja mais uma modalidade de serviço de saúde entre outros afinal, não é um serviço descolado da RAPS -, situam-se valores clínicos nesse serviço que são singulares, na medida que se espera estar melhor capacitado para o cuidado de casos graves.

Nessa perspectiva, tratando-se de serviços públicos de saúde, além dos valores clínicos também se figuram os valores políticos. Para Elia (2005), se o dispositivo CAPS é o espaço que corporifica uma determinada lógica do cuidado em um território - lógica que é regida pelas diretrizes públicas de saúde mental -, esse equipamento terá, dentre as principais tarefas, "a de articular-se aos ambulatórios do território em questão, sob pena de não cumprir seu mandato político-social de fazer valer tais diretrizes. Isso não apenas pressupõe uma rede ambulatorial, mas exige que o CAPS a ela se articule" (p. 57). Nesse sentido, entende-se que o CAPSi seria tanto um espaço clínico criado para o cuidado e tratamento de crianças e adolescentes em sofrimento psíquico grave, quanto o dispositivo responsável por ordenar a demanda em SMCA, fazendo valer a política do cuidado em rede e no território. Em termos práticos, Elia (2005) afirma:

O CAPSi vai acolher universalmente todo aquele que o procurar, vai problematizar a demanda que lhe chega, vai absorver algumas delas sob a forma de cuidado dispensado no próprio CAPSi, vai encaminhar outros tantos, acompanhando o encaminhamento como um ato seu, pelo qual é responsável, vai convocar vários setores e instâncias do território (intersetorialidade), além de outros equipamentos de saúde mental e de saúde, vai chamar a rede educacional, assistencial, jurídica, enfim, vai operar a ordenação da rede, sem com isso recorrer a qualquer tipo de ato que se superponha, confunda ou nivele com os atos da gestão. (p. 57)

Destaca-se a advertência do autor para que o ato político do CAPSi não seja confundido com atos de gestão. Isso significa dizer que não deve ser depositada no CAPSi a tarefa administrativa, posto que isso faria o serviço ocupar um lugar hierárquico no poder, no sentido foucaultiano 
do termo, isto é, no campo político de governo das ações e do domínio do saber. Pelo contrário, o ato político do CAPSi é o de fazer valer o compartilhamento em rede, ou seja, a lógica intersetorial e o saber compartilhado, e isso significa exercer a clínica ampliada em sua essência. "É a própria clínica exercida sob a forma lógica da intervenção ampliada que funda o ato do CAPSi” (Elia, 2005, p. 57). Nesses termos, entendemos que "o ato do CAPSi" que o autor se refere, a saber, sua dimensão política, é, em sua essência, uma dimensão clínica orientada pelo paradigma da atenção psicossocial.

\section{Considerações finais}

Retoma-se que o objetivo deste estudo foi caracterizar o CAPSi de Vitória em seu décimo ano de funcionamento e comparar com os achados da caracterização do primeiro ano de funcionamento. Entre os principais achados, destacam-se:

1. O aumento do número de crianças e adolescentes do sexo feminino endereçadas ao CAPSi, cuja marca são as queixas de automutilação e práticas de cutting que, por sua vez, denotam um sofrimento psíquico possível de ser escutado como sinal de risco de suicídio ou índice de alguma psicopatologia, questões que exigem complexidade no cuidado.

2. A prevalência de usuários do sexo masculino no CAPSi, cuja marca são os problemas externalizantes, tais como comportamentos agressivos, hiperatividade e uso de álcool e outras drogas. Esses achados se mantêm como um denominador comum entre os estudos de caracterização da população assistida pelos CAPSi.

3. Novamente, identificou-se baixa prevalência de quadros que figuram psicoses e autismos, fato que deve ser constantemente questionado, posto que crianças e adolescentes de estrutura psicótica e autista constituem clientela prioritária do CAPSi.

4. Se por um lado se mantém a baixa prevalência de usuários de estrutura psicótica e autista, prevalecem os usuários com questões que envolvem dificuldades nos espaços escolares, como hiperatividade, agressividade e desatenção. Esses casos tendem a ser endereçados ao
CAPSi por hipóteses de que o déficit no espaço escolar possa ser índice de transtornos no desenvolvimento, o que em muitos casos não se confirma, por isso a contrarreferência do caso para unidades de menor complexidade. Destaca-se, portanto, a necessidade de práticas que promovam ações corresponsáveis entre CAPSi, UBS e instituições escolares nas ações de cuidado em SMCA.

5. A prescrição de medicamentos psiquiátricos para crianças e adolescentes sem registro ou formulação de alguma hipótese diagnostica, o que tende apontar para a dificuldade de se situar um diagnóstico ético e um PTS que não esteja intimamente ligada à resposta medicamentosa. Como ressaltado, essas práticas são antagônicas ao modelo psicossocial, posto que implicam tomar, de saída, queixas sobre o usuário como a demanda de tratamento, o que propende barrar qualquer possibilidade de o sujeito participar e se envolver com seu sofrimento e seu cuidado.

6. A ausência de informações sobre a escolaridade dos usuários em $26,65 \%$ dos prontuários e sobre a procedência dos encaminhamentos em 22,35\% dos casos em atendimento regular. Para um serviço que tem mandato público de ordenação da demanda em SMCA, essas ausências de informações dificultam o desenvolvimento de ações que considerem os aspectos psicossociais da população assistida.

Diante dos dados, o estudo aponta para a necessidade de maior investimento em apoio matricial do CAPSi para com as UBS, de forma que discutam e planejem estratégias de cuidado a serem tomadas em conjunto, no intuito de aumentarem a efetividade de suas ações psicossociais. Do mesmo modo, entendemos ser necessário ampliar ações de orientação à população sobre os serviços prestados pelo CAPSi, posto que o quantitativo atendido pelo serviço ainda é relativamente baixo.

De maneira geral, entendemos que as dificuldades enfrentadas pelo serviço remontam os desafios de se exercer a clínica ampliada e a lógica do trabalho psicossocial. Nesses termos, parece-nos importante, também, o serviço investir em supervisão clínico-institucional, de forma que não fique encapsulado em si mesmo e com dificuldade de ter uma inserção mais ampla no território e práticas clínicas com potencial emancipatório.

\section{0 years of CAPSi: comparing the characterization of users served}

Abstract: This descriptive retrospective study used medical records to characterize children and adolescents cared in a CAPSi during their tenth year of operation and to compare with an analogous study carried out in the first year of operation. 
Data were analyzed using SPSS and IRaMuTeQ. There was an increase of $51.85 \%(n=84)$ in demand compared to the first year. There was an increase in female users, whose complaints concerned self-mutilation. However, the prevalence of male users remained, whose complaints concerned behavioral issues such as aggression and hyperactivity. In this study, we discuss the articulations between CAPSi and other services of the Psychosocial Care Network, especially regarding referrals. It also discusses medicalization without record of prognosis and the challenges of exercising the "expanded clinical" practice and the logic of psychosocial work, which opposes the exclusively biomedical paradigm, simplifying the phenomena of child development.

Keywords: mental health, health services, child, teenager.

\section{0 ans d'une CAPSi : comparaison de la caractérisation des utilisateurs du service}

Résumé: Une étude rétrospective descriptive. L'objectif c'était de caractériser les enfants et adolescents traités dans une CAPSi au cours de leur dixième année d'activité et faire la comparaison avec l'étude analogue réalisée au long de la première année. Les données ont été analysées à l'aide des logiciels SPSS et IRaMuTeQ. II a été identifié une augmentation de 51,85\% $(n=84)$ de la demande par rapport à la première année. Il y a eu une augmentation du nombre d'utilisateurs du sexe féminin, dont la marque était des plaintes d'automutilation. Cependant, la prévalence des utilisateurs du sexe masculin est restée, dont la marque était des problèmes de comportement, comme l'agressivité et l'hyperactivité. Dans cette étude, nous discutons des questions relatives aux articulations entre le CAPSi et les autres services du réseau de soins psychosociaux, particulièrement en ce qui concerne les renvois. II aborde également la médicalisation sans enregistrement d'hypothèses diagnostiques et les défis de l'exercice de la "clinique élargie" et à la logique psychosocial, qui s'oppose au paradigme exclusivement biomédical simplifiant les phénomènes de développement de l'enfant.

Mots-clés : Santé mentale, Services de santé, Enfant, Adolescent.

\section{0 años de un CAPSi: comparación de la caracterización de los usuarios atendidos}

Resumen: Estudio retrospectivo, de carácter descriptivo. Por medio de recolección de datos en historiales, se objetivó caracterizar a niños y adolescentes atendidos en un CAPSi durante su décimo año de funcionamiento, y comparar los datos con un estudio análogo realizado en su primer año de funcionamiento. Para el análisis de datos, se utilizó los softwares SPSS e Iramuteq. Se identificó un aumento del 51,85\% ( $n=84)$ de la demanda en relación al primer año. Se observó un aumento de usuarios del sexo femenino, cuya marca fueron quejas de automutilación. Sin embargo, se mantuvo la prevalencia de usuarios del sexo masculino, cuya marca fueron problemas de comportamiento, como agresividad e hiperactividad. En este estudio, se discuten las articulaciones entre CAPSi y otros servicios de la Red de Atención Psicosocial, especialmente sobre las remisiones. También se discute la medicalización sin registro de hipótesis diagnósticas y los desafíos de ejercer la clínica extendida y la lógica del trabajo psicosocial, que se opone al paradigma exclusivamente biomédico simplificador de los fenómenos del desarrollo infantil.

Palabras clave: salud mental, servicios de salud, niño, adolescente.

\section{Referências}

Alberti, S., \& Figueiredo, A. C. (2006). Psicanálise e saúde mental: uma aposta. Rio de Janeiro, RJ: Companhia de Freud.

Amarante, P. (1995). Novos sujeitos, novos direitos: o debate em torno da reforma psiquiátrica. Cadernos de Saúde Pública, 11(3), 491-494.

Avellar, L. Z., \& Safra, G. (2018). Saúde mental infantojuvenil: contribuição de alguns conceitos da obra de D. W. Winniccott para a constituição de serviços de assistência In: I. Ferrari \& J. Ferreira-Neto (Orgs.), Politicas públicas e clínica: estudos em psicologia e psicanálise (pp. 134154). Belo Horizonte, MG: Editora PUC Minas.
Beltrame, M. M., \& Boarini, M. L. (2013). Saúde mental e infância: reflexões sobre a demanda escolar de um CAPSi. Psicologia: Ciência e Profissão, 33(2), 336-349.

Bernardes, S. M. (2015). Tornar-se (in) visivel: um estudo na rede de atenção psicossocial de adolescentes que se automutilam (Dissertação de Mestrado). Universidade Federal de Santa Catarina, Santa Catarina, SC.

Bueno, A. R. (2013). Terapia Ocupacional no campo da saúde mental infanto-juvenil: revelando as ações junto aos Centros de Atenção Psicossocial Infanto-juvenil (Dissertação de Mestrado). Universidade Federal de São Carlos, São Carlos, SP. 
Camargo, B. V, \& Justo, A. M. (2013). IRAMUTEQ: um software gratuito para análise de dados textuais. Temas em psicologia, 21(2), 513-518.

Canguilhem, G. (2002). O normal e o patológico (5a ed., rev. e aum.). Rio de Janeiro, RJ: Forense Universitária. (Trabalho original publicado em 1943)

Capsi é centro de Atenção Psicossocial Infanto-juvenil. (2019). Vitória, ES: Prefeitura Municipal de Vitória. Recuperado de http://bit.ly/3cfBntv

Carvalho, I. L. (2012). Uso de psicofármacos em adolescentes atendidos pelos centros de atenção psicossocial infantojuvenil de Fortaleza-CE (Dissertação de Mestrado). Universidade de Fortaleza, Fortaleza, CE.

Carvalho, I. L., Gondim, A. P., Holanda, T. T., \& Alencar, V. P. (2014). CAPSi: avanços e desafios após uma década de funcionamento. Cadernos Brasileiros de Saúde Mental, 6(14), 42-60.

Cervo, M. D. R., \& Silva, R. D. (2014). Um olhar sobre a patologização da infância a partir do CAPSI. Revista Subjetividades, 14(3), 442-453.

Cirino, O. (2004). Apresentação. In: T. Ferreira (Org.), $A$ criança e a saúde mental: enlaces entre a clínica e a politica (pp. 9-12). Belo Horizonte, MG: Autêntica.

Couto, M. C., \& Delgado, P. G. (2015). Crianças e adolescentes na agenda política da saúde mental brasileira: inclusão tardia, desafios atuais. Psicologia Clínica, 27(1), 17-40.

Couto, M. C., Duarte, C. S., \& Delgado, P. G. (2008). A saúde mental infantil na Saúde Pública brasileira: situação atual e desafios. Revista Brasileira de Psiquiatria, 30(4), 384-389.

D’Abreu, L. C. (2012). O desafio do diagnóstico psiquiátrico na criança. Contextos Clínicos, 5(1), 2-9.

Daltro, M. C., Moraes, J., \& Marsiglia, R. G. (2018). Caregivers of children and adolescents with mental disorders: social, family and sexual changes. Saúde $e$ Sociedade, 27(2), 544-555.

Delfini, P. S., Dombi-Barbosa, C., Fonseca, F. L., Tavares, C. M., \& Reis, A. O. (2009). Perfil dos usuários de um centro de atenção psicossocial infantojuvenil da grande São Paulo, Brasil. Revista Brasileira de Crescimento e Desenvolvimento Humano, 19(2), 226-236.

Elia, L. (2005). A Rede da Atenção na Saúde Mental: articulações entre CAPS e ambulatórios. In: Ministério da Saúde (Ed.), Caminhos para uma política de saúde mental infanto-juvenil (2a ed., rev., pp. 45-57). Brasília, DF: Editora MS.

Faria, M. R. (2016). Introdução à psicanálise de crianças: o lugar dos pais. São Paulo, SP: Toro Editora.

Furtado, J. P., \& Onocko-Campos, R. (2005). A transposição das políticas de saúde mental no Brasil para a prática nos novos serviços. Revista Latinoamericana de Psicopatologia Fundamental, 8(1), 109-122.

Hoffmann, M. C., Santos, D. N., \& Mota, E. L. (2008). Caracterização dos usuários e dos serviços prestados por Centros de Atenção Psicossocial Infanto-Juvenil. Cadernos de Saúde Pública, 24(3), 633-642.
Jerusalinsky, J. (2018). Detecção precoce de sofrimento psíquico versus patologização da primeira infância: face à lei $\mathrm{n}^{\circ} 13.438 / 17$, referente ao estatuto da criança e do adolescente. Estilos da Clínica, 23(1), 83-99.

Lei $n^{\circ} 10.216$, de 6 de abril de 2001 (2001). Dispõe sobre a proteção e os direitos das pessoas portadoras de transtornos mentais e redireciona o modelo assistencial em saúde mental. Brasília, DF: Presidência da República. Recuperado de http://bit.ly/2HSYfRP

Leitão, I. B., Constantinidis, T. C., \& Avellar, L. Z. (2019). Produção de conhecimentos sobre o CAPSI entre 2002 e 2017: revisão integrativa da literatura. Estudos Interdisciplinares em Psicologia, 10(3), 181-205.

Lima, R. C. (2005). Somos todos desatentos? O TDA/H e a construcão de bioidentidades. Rio de Janeiro, RJ: Relume Dumará.

Luckow, H. I., \& Cordeiro, A. M. (2017). Concepções de adolescência e educação na atuação de profissionais do CAPSi. Psicologia: Ciência e Profissão, 37(2), 393-403.

Machado, C. M., Luiz, A. M., Marques Filho, A. B., Miyazaki, M. C., Domingos, N. A., \& Cabrera, E. M. (2014). Ambulatório de psiquiatria infantil: prevalência de transtornos mentais em crianças e adolescentes. Psicologia: Teoria e Prática, 16(2), 53-62.

Ministério da Saúde (2003). Saúde mental e atenção básica: o vínculo e o diálogo necessários. Brasília, DF: Editora MS. Recuperado de http://bit.ly/2SHJ2ZP

Ministério da Saúde (2005). Caminhos para uma política de saúde mental infanto-juvenil. Brasília, DF: Editora MS. Recuperado de http://bit.ly/3bThxnz

Ministério da Saúde (2010). Diretrizes nacionais para a atenção integral à saúde de adolescentes e jovens na promoção, proteção e recuperação da saúde. Brasília, DF: Ministério da Saúde. Recuperado de http://bit.ly/37JpsAE

Ministério da Saúde (2013). Estratégias para o cuidado da pessoa com doença crônica: diabetes mellitus. Brasília, DF: Ministério da Saúde. Recuperado de http://bit.ly/2PaNjmq

Ministério da Saúde (2014a). Atenção psicossocial a crianças e adolescentes no SUS: tecendo redes para garantir direitos. Brasília, DF: Ministério da Saúde. Recuperado de http://bit.ly/37LMN4T

Ministério da Saúde (2014b). Fórum Nacional de Saúde Mental Infantojuvenil: recomendações de 2005 a 2012. Brasília, DF: Ministério da Saúde. Recuperado de http:// bit.ly/2PaVYVP

Monteiro, A. R., Teixeira, L. A., Silva, R. S., Rabelo, K. S., Tavares, S. V., \& Távora, R. O. (2012). Sofrimento psíquico em crianças e adolescentes: a busca pelo tratamento. Escola Anna Nery, 16(3), 523-529.

Muylaert, C. J., Delfini, P. S., \& Reis, A. O. A. (2015). Relações de gênero entre familiares cuidadores de crianças e adolescentes de serviços de saúde mental. Physis: Revista de Saúde Coletiva, 25(1), 41-58.

Onocko-Campos, R. (2001). Clínica: a palavra negada sobre as práticas clínicas nos serviços substitutivos de saúde mental. Saúde em Debate, 25(58), 98-111. 
Portaria $n^{\circ}$ 336, de 19 de fevereiro de 2002 (2002). Brasília, DF: Ministério da Saúde. Recuperado de http://bit.ly/39WA5BT

Portaria n $^{\mathrm{o}} 3.088$, de 23 de dezembro de 2011 (2011). Institui a Rede de Atenção Psicossocial para pessoas com sofrimento ou transtorno mental e com necessidades decorrentes do uso de crack, álcool e outras drogas, no âmbito do Sistema Único de Saúde (SUS). Brasília, DF: Ministério da Saúde. Recuperado de http://bit.ly/32cyC7C

Quintanilha, B. C., Belotti, M., Tristão, K. G., \& Avellar, L. Z. (2017). A produção do cuidado em um centro de atenção psicossocial infantojuvenil. Mental, 11(20), 261-278.

Reis, A. O., Delfini, P. S., Dombi-Barbosa, C., \& Oliveira, M. F. (2010). Crianças e adolescentes em sofrimento psíquico atendidos nos centros de atenção psicossocial infantojuvenis. In: E. Lauridsen-Ribeiro \& O. Tanaka (Orgs.), Atenção em Saúde Mental para crianças e adolescentes no SUS (pp. 186-210). São Paulo, SP: Hucitec.

Ronchi, J. P., \& Avellar, L. Z. (2010). Saúde mental da criança e do adolescente: a experiência do CAPSi da cidade de Vitória-ES. Psicologia: Teoria e Prática, 12(1), 71-84.

Ronchi, J. P., \& Avellar, L. Z. (2013). Ambiência na Atenção Psicossocial Infanto-juvenil: um estudo no CAPSi. Saúde e Sociedade, 22(4), 1045-1058.

Salvador, D., \& Pio, D. (2016). Apoio Matricial e CAPSi: desafios do cenário na implantação do matriciamento em saúde mental. Saúde em Debate, 40(111), 246-256.

Scarcelli, I. R., \& Alencar, S. L. (2009). Saúde mental e saúde coletiva: intersetorialidade e participação em debate. Cadernos Brasileiros de Saúde Mental, 1(1), 67-76.

Silva, A. C., \& Botti, N. L. (2017). Comportamento autolesivo ao longo do ciclo vital: Revisão integrativa da literatura. Revista Portuguesa de Enfermagem de Saúde Mental, (18), 67-76.

Silva, H., \& Ghazzi, M. (2016). Diagnóstico na infância: quais as implicações possíveis? Interação em Psicologia, 20(2).
Silva, J. R., Guazina, F. M., Pizzinato, A., \& Rocha, K. B. (2019). O "singular" do projeto terapêutico: (im) possibilidades de construções no CAPSi. Revista Polis e Psique, 9(1), 127-146.

Silva Júnior, A. G., Merhy, E. E., \& Carvalho, L. C. (2003). Refletindo sobre o ato de cuidar da saúde. In: R. E. Pinheiro \& R. A. Mattos (Org.), Construção da integralidade: cotidiano, saberes e práticas em saúde (pp. 113-128). Rio de Janeiro, RJ: Abrasco.

Taño, B. L., \& Matsukura, T. S. (2015). Saúde mental infanto-juvenil e desafios do campo: reflexões a partir do percurso histórico. Cadernos de Terapia Ocupacional da UFSCar, 23(2), 439-447.

Teixeira, M. R., Couto, M. C., \& Delgado, P. G. (2017). Atenção básica e cuidado colaborativo na atenção psicossocial de crianças e adolescentes: facilitadores e barreiras. Ciência \& Saúde Coletiva, 22(6), 1933-1942.

Teixeira, R. M, \& Jucá, V. J. (2014). Caracterização dos usuários de um centro de atenção psicossocial infantojuvenil do município de Salvador (BA). Revista de Psicologia, 5(2), 70-84.

Tristão, K. G. (2018). CAPSij como lugar de cuidado para crianças e adolescentes em uso de substâncias psicoativas (Tese de Doutorado). Universidade Federal do Espírito Santo, Vitória, ES.

Vanoli, E. N., \& Bernardino, L. F. (2008). Psicose infantil: uma reflexão sobre a relevância da intervenção psicanalítica. Estilos da Clínica, 13(25), 250-267.

Recebido: $29 / 01 / 2019$

Revisado: 06/05/2019

Revisado: 02/10/2019

Aprovado: 31/01/2020 